\title{
EMPRENDIMIENTO FEMENINO EN MÉXICO: FACTORES RELEVANTES PARA SU CREACIÓN Y PERMANENCIA
}

FEMALE ENTREPRENEURSHIP IN MÉXICO: RELEVANT FACTORS FOR THEIR CREATION AND PERMANENCE

\section{EMPREENDEDOR FEMININO NO MÉXICO: FATORES RELEVANTES PARA SUA CRIAÇÃO E PERMANÊNCIA}

\section{PAZ CALDERÓN_Yannet, ESPINOSA ESPÍNDOLA_Mónica Teresa}

Doctora en Economía Política del Desarrollo por la Benemérita Universidad Autónoma de Puebla, México, Profesora Investigadora de la Universidad Tecnológica de la Mixteca. Email: ypaz@mixteco.utm.mx, México.

Doctora en Administración por la Universidad Autónoma de Querétaro, México, Profesora Investigadora de la Universidad Tecnológica de la Mixteca. Email: monitte2005@hotmail.com, México.

Recibido: 17 de noviembre de 2019

Aprobado: 20 de diciembre de 2019

DOI: https://doi.org/10.22267/rtend.192002.117

\section{RESUMEN}

El objetivo de este documento es identificar los factores más relevantes que contribuyen a la creación y permanencia de empresas encabezadas por mujeres en México, para ello se define el 
perfil de las mujeres que inician un negocio y las condiciones en las que se suman a esta actividad.

La investigación es descriptiva basada en fuentes estadísticas y documentales, se revisan datos del Instituto Nacional de Estadística y Geografía (INEGI), de la Secretaria del Trabajo y Previsión Social (STPS) y del Instituto Nacional de las Mujeres (INMUJERES). Los resultados muestran, que las mujeres se ha visto afectadas de manera severa por el desempleo y la precariedad del mercado de trabajo, que el emprendimiento femenino surge, principalmente como una manera de hacer frente a la pobreza y marginación de sus familias y no como una forma de hacer uso productivo de recursos materiales, físicos e intelectuales. Por ello, es importante que en la construcción de políticas públicas se consideran las condiciones, las necesidades y las motivaciones que tienen las mujeres para crear sus propias empresas, para que los apoyos gubernamentales impulsen la sobrevivencia y el crecimiento de estos emprendimientos.

Palabras Clave: emprendimiento, mujeres, empleo, participación en la fuerza laboral, política pública, trabajo femenino

JEL: J23, J16, J2, J22, J18, J21

\begin{abstract}
ABSTRAC
The objective of this document is to identify the most relevant factors that contribute to the creation and permanence of women-led companies in Mexico, for this the profile of women who start a business and the conditions in which they join this activity are defined. The research is descriptive, based on statistical and documentary sources, data from the National Institute of Statistics and Geography (INEGI), the Ministry of Labor and Social Welfare (STPS) and the National Women's Institute (INMUJERES) are reviewed. The results show that women have been severely affected by unemployment and the precariousness of the labor market, that female entrepreneurship arises, mainly as a way to face the poverty and marginalization of their families and not as a way of make productive use of material, physical and intellectual resources. Therefore, it is important to consider the conditions, needs and motivations in the construction of public policies for women to create their own businesses, so that government support promotes the survival and growth of these ventures.
\end{abstract}


Keywords: entrepreneurship, women, employment, labor force participation, public policy, female labor

JEL: J23, J16, J2, J22, J18, J21

\section{RESUMO}

O objetivo deste documento é identificar os fatores mais relevantes que contribuem para a criação e permanência de empresas lideradas por mulheres no México, para isso são definidos o perfil das mulheres que iniciam um negócio e as condições em que ingressam nessa atividade. A pesquisa é descritiva, com base em fontes estatísticas e documentais, dados da Instituto Nacional de Estatística e Geografia (INEGI), Ministério do Trabalho e Bem-Estar Social (Secretaria del Trabajo y Previsión Social -STPS) e o Instituto Nacional da Mulher (Instituto Nacional de las Mujeres- INMUJERES). Os resultados mostram que as mulheres foram severamente afetadas pelo desemprego e pela precariedade do mercado de trabalho, que o empreendedorismo feminino surge, principalmente como forma de lidar com a pobreza e a marginalização de suas famílias e não como maneira de fazer uso produtivo de recursos materiais, físicos e intelectuais. Portanto, é importante que, na construção de políticas públicas, sejam consideradas as condições, necessidades e motivações que as mulheres têm para criar seus próprios negócios, para que o apoio do governo conduza à sobrevivência e ao crescimento dessas empresas.

Palavras-Chave: empreendedorismo, mulheres, emprego, participação da força de trabalho, políticas públicas, trabalho feminino

JEL: J23, J16, J2, J22, J18, J21

\section{INTRODUCCIÓN}

En México para el segundo trimestre de 2019 la tasa de desempleo fue de 3.3\%, además el mercado laboral presenta altos nivele de informalidad, el 56.9\% de la Población Económicamente Activa (PEA) se encuentra en esta condición, por lo que se requiere impulsar y desarrollar actividades productivas que puedan contribuir a la generación de fuentes de trabajo. De acuerdo a la Encuesta Nacional sobre Productividad y Competitividad de las Micro, Pequeñas 
y Medianas Empresas (ENAPROCE) que llevó a cabo el Instituto Nacional de Estadística y Geografía (INEGI) por primera vez durante los meses de junio y julio de 2015, existen en el país 4 millones de empresas: 3.9 millones de tamaño micro, 79 mil pequeñas, 16 mil medianas y casi 11 mil empresas grandes. Las micro, pequeñas y medianas empresas (MIPYMES) generan el 63\% del empleo y el 35\% del Producto Interno Bruto (PIB).

A pesar de la importancia económica de estas unidades de negocio, presentan bajos niveles de productividad. Son diversos los factores que explican este fenómeno en las MIPYMES, sin embargo la mayoría de los dueños de este tipo de empresas inician sus negocios con pocos conocimientos y habilidades relacionados con administración, contabilidad, mercadotecnia, estudios de mercados y financiamiento, lo que provoca que su esperanza de vida sea corta. No cuenta con registros formales o informales de sus actividades, todo lo aprenden en base a la experiencia que van acumulando a base de prueba y error. Esto hace evidente la necesidad de apoyar el emprendimiento a través de las instituciones educativas, en todos sus niveles, de las incubadoras, de organismos gubernamentales y de organizaciones privadas y de la sociedad civil, entre otros.

En el caso particular de las mujeres y su relación con la creación de negocios este es un tema de suma importancia, principalmente en países en desarrollo, los Objetivos de Desarrollo Sostenible contemplan el empoderamiento de las mujeres para que puedan mejorar las condiciones de su participación en la política, la economía, la cultura, los negocios y en todas las áreas del desarrollo, con ello se podrán mejorar las condiciones de vida de sus familias y sus comunidades. Esto es muy relevante debido a que la población femenina enfrenta diversos tipos de discriminación, lo que repercute en el ejercicio pleno de sus derechos. Además se requiere de la generación de empleos productivos y decentes, incentivar el emprendimiento y la innovación. La incorporación de la mujer al trabajo remunerado y formal puede contribuir de manera importante en diversas áreas de su quehacer cotidiano, además de su contribución a la actividad económica del país, impactaría de manera positiva en la reducción de la pobreza y al mejoramiento de sus derechos e independencia, ellas son más proclives a destinar sus ingresos a satisfacer necesidades de su hogar y de su comunidad como educación, salud y vivienda, (CEPAL, 2018a; CEPAL, 2018b; MUJERES ONU, 2016) 
Las mujeres mexicanas aún enfrentan importantes dificultades para poder participar e insertarse plenamente en el mercado laboral, el rol de género que les impone la responsabilidad de hacerse cargo del trabajo doméstico no remunerado, lo cual complica que puedan coordinar el empleo fuera de casa y la atención de su vida familiar. La prevalencia de las desigualdades entre hombres y mujeres en ámbitos como el político, el económico y el social, evita que puedan involucrarse de lleno en actividades productivas.

De esta manera, el objetivo del documento es identificar los factores más importantes que motivan a las mujeres mexicanas para iniciar un negocio, para ello se exploran las condiciones en las que se incorporan a esta actividad. Esto permitió observar las necesidades y los retos que este tipo de empresas enfrentan y que, a través de las políticas públicas pueden atenderse los problemas que enfrentan y así sus empresas puedan sobrevivir y crecer.

La vía analítica que se sigue en este trabajo consta de cinco apartados, en el primero se revisa, a través de diversos autores, los conceptos de emprendimiento y emprendimiento femenino. En el segundo se explica la metodología que se siguió para lograr el objetivo del documento. En el tercero se presentan las condiciones de las mujeres en el mercado de trabajo, se describe el perfil de las mujeres emprendedoras y se analizan los principales elementos que pueden apoyar el impulso a los emprendimientos femeninos. En el último, se presentan las reflexiones finales.

\section{EMPRENDIMIENTO FEMENINO}

Existen diversas definiciones del término emprendedor, todas coinciden en que es una persona que decide poner en práctica una idea que la lleva a iniciar una empresa o negocio, las motivaciones suelen ser de diversa índole, pero lo que busca es materializar sus inquietudes empresariales que desembocaran en llevar al mercado un bien o servicios. Amaru, (2008:1) señala que "la palabra emprendedor proviene del latín imprendere, que significa tomar la decisión de realizar una tarea difícil y laboriosa, poner en ejecución”. En el mismo sentido Pierre \& Molina (2012) retoman la definición emitida en el 2003 por la Organización para la Cooperación y el Desarrollo Económico (OCDE) que indica que la actividad emprendedora está relacionada con el riesgo, la creatividad y la innovación y pone especial énfasis en este último elemento, ya que considera que dentro de los que inician un negocio no se deben considerar aquellos que solo imitan algún tipo producto ni los que compran empresas, esto tendría sus salvedades porque habría que considerar las condiciones y circunstancias dentro de la cuales 
puedan rugir estas formas de emprendimiento, una persona podría comprar una empresas para desarrollarla por medio de diversas mejoras en distintas áreas de la misma. Por lo tanto, se puede decir que la persona que decide emplear recursos para apoyar la creación de nuevas empresas debe ser poco adverso al riesgo para poder tomar decisiones en momentos de incertidumbre.

Los emprendimientos pueden ser de diversa índole como: autoempleos o emprendimiento por necesidad, emprendimientos corporativos, emprendimientos en familia, emprendimientos sociales, (Vallmitjana, 2014); los cuales dependerán de los recursos con los que dispongan los individuos así como de las necesidades que tengan de obtener ingresos.

Existen ciertos factores exógenos que se relaciona con el contexto o entorno económico nacional e incluso mundial, que permiten el desarrollo de la actividad emprendedora, que de acuerdo con Vallmitjana (2014), son los siguientes:

a) contexto macroeconómico

b) acceso a fuentes de financiación

c) contexto social

d) contexto tecnológico

e) políticas de soporte de los gobiernos

Se puede observar que el elemento que atraviesa a todos ellos es la política pública que desde sus diferentes espacios de acción afecta -positiva o negativamente- a los emprendedores.

En cuanto a las motivaciones que tienen las personas para comenzar un negocio existen dos que son las más comunes, la primera es la detección de un área de oportunidad de mercado y la segunda se relaciona con la necesidad de autoemplearse como alternativa a la falta de trabajo, (Valls, Cruz, Torruella, Juanes, Canessa, \& Hormiga, 2012; Ruiz, Camelo \& Leal, 2015).

Canales, Román \& Ovando (2017), definen las características sociodemográficas de la población joven emprendedora en México y encuentran que los hombres casados y jefes de familia son más propensos a participar en la creación de empresas, en el caso de las mujeres ellas tiene mayor propensión hacia el emprendimiento después de pasar por una separación conyugal, lo que llevaría a decir que lo hacen por necesidad de obtener ingresos, lo que se refuerza con otro de los resultados encontrados en este mismo trabajo: que a mayor edad, las mujeres se inclinan más por el emprendimiento. 
Además es innegable que los en los últimos años los empleos formales han ido disminuyendo en todo el mundo, lo que ha provocado que la generación de ingresos por parte de la mujer sea cada vez más importante para la supervivencia de los hogares. Sin embargo, las empresas iniciadas por mujeres se encuentran normalmente en el sector de servicios, comercio, hoteles y restaurantes, principalmente en la áreas urbanas, lo que muestra una prolongación de las actividades que realizan en sus hogares como preparación de alimentos, aseo, cuidado de niños y ancianos y educativas, se puede observar que ellas en el ámbito del trabajo remunerado siguen vinculadas a su rol de género.

Asimismo, las mujeres enfrentan diferentes obstáculos al iniciar sus negocios, entre los que se pueden destacar: a) poca confianza en sí mismas, b) social y culturalmente se les relaciona con las labores del hogar, por lo que terceras personas no consideran una buena opciones hacer negocios con ellas, y c) los bajos niveles educativos que en promedio tiene la población femenina provoca que esto sea una barrera cuando ellas deciden administrar su propia empresa, (Viadana, Zubeldía, Tomarelli \& Morales, 2016).

En una investigación realizada por Cisneros, Meza \& Meza, (2016) en una zona marginal de la ciudad Guayaquil, Ecuador encontraron que las mujeres de ese lugar optan por los emprendimientos que les permiten seguir cuidando de su hogar y sus hijos, es decir que nos les implique desplazamiento, ya que ella siguen teniendo como prioridad y necesidad seguir con su rol de género. También hallaron que debido a sus bajos niveles educativos tienen problemas con el manejo de las financias de sus negocios y en general todo lo relacionado con temas relacionados con riesgos y administración de las empresas.

\section{METODOLOGÍA}

Es un estudio descriptivo que "buscan especificar las propiedades, las características y los perfiles de personas, grupos, comunidades, procesos, objetos o cualquier otro fenómeno que se someta a un análisis" (Hernández, Fernández \& Baptista (2006:80). En esta investigación se recopilo información para identificar los factores más relevantes que contribuyen a la creación y permanencia de empresas encabezadas por mujeres en México, para ello se incorporaron elementos teóricos y cuantitativos. En la primera parte de este este trabajo se realizó una revisión 
exhaustiva de la bibliografía existente sobre emprendimiento y emprendimiento femenino. En la segunda, se siguió un esquema analítico a partir de información estadística en base a la recuperación y análisis de datos del Instituto Nacional de Estadística y Geografía (INEGI), específicamente la Cuenta Satélite del Trabajo No Remunerado de los Hogares de México, 2017, la cual proporcionar información sobre el valor económico de tiempo que se utiliza en la producción de bienes y servicios relacionados con los quehaceres domésticos y de cuidado que realizan los miembros de un hogar para la satisfacción de las necesidades del mismo y por el que no reciben ninguna remuneración, se tomaron estadísticas referentes a la población femenina mayor a 12 años. También se utilizaron cifras de la de la Secretaria del Trabajo y Previsión Social (STPS) sobre las condiciones laborales de la PEA para el segundo trimestre de 2019. Del Instituto Nacional de las Mujeres (INMUJERES) se tomaron datos de las principales características socioeconómicas de las mujeres empleadoras en el país para 2019, esto permitió definir el perfil de las mujeres empresarias.

\section{RESULTADOS Y DISCUSIÓN}

El mercado de trabajo es un espacio donde también se pueden observar las diferencias entre hombres y mujeres, analizarlo permite identificar elementos que ayuden al diseño de políticas que apoyen una mejor incorporación de la población femenina a las actividades productivas. En este apartado se hace una exploración de las características de las condiciones laborales que enfrentan las mujeres y posteriormente se define el perfil de las mujeres emprendedoras.

\section{Condiciones laborales de la población}

En México el mercado laboral presenta importantes niveles de precariedad e informalidad, para el segundo trimestre de 2019, la tasa de desempleo fue de 3.3\% para la población en general, siendo los jóvenes y las mujeres jóvenes quienes tienen mayor dificultad para encontrar un trabajo. La mayor parte de los desempleados se encuentran entre los 20 y 29 años, y tienen primaria y educación superior, principalmente. La mayor parte de las personas que cuenta con empleo tiene secundaria o educación media superior (STPS, 2019). El mercado laboral mexicano está caracterizado por la informalidad, el 56.9\% de la PEA se encuentra en esta condición, además aquellos que están ocupados son en su mayoría asalariados (68\%), empleadores apenas el $5 \%$, otro cifra que muestra las condiciones de precariedad de los empleados es la tasa crítica 
de ocupación que oscila alrededor del 19.3\%, lo que indica que ese porcentaje de la población recibe un salario menor al mínimo por trabajar más de 48 horas y únicamente el 55\% de los trabajadores cuenta con seguridad social, (figura 1), (STPS, 2019).

\section{Las mujeres en el mercado laboral}

Para el segundo trimestre de 2019, la tasa neta de participación femenina en el mercado de trabajo es de $43.7 \%$ mucho menor que la de los hombres que es de $76.9 \%$, y además se encuentran ocupadas, en su mayoría en la informalidad, reciben bajos salarios y enfrentan una mayor tasa de desempleo que los hombres (STPS, 2019). Una característica de esta población y que en parte explica su baja participación en el mercado de trabajo, en comparación con la de los hombres, es que dedican parte de su tiempo a atender actividades relacionadas con el cuidado del hogar, en promedio contribuyeron en el 2017, con el $74.6 \%$ del valor del trabajo no remunerado relacionado con alimentación; limpieza y cuidado de la ropa, el calzado y la vivienda; cuidados y apoyo, compras y administración del hogar (figura 2), (INEGI, 2017). Es innegable que la población trabajadora femenina tienen importantes desventajas al incorporarse a las actividades productivas, las cuales tienen su fundamento en el bajo nivel educativo y en la prevalencia del rol de género, que la ubica como las principales cuidadoras del hogar, lo que provoca que laboren principalmente en el sector servicios y de manera subordinada. 


\section{La Población Económicamente Activa: 56,038,471}

Hombres $61.4 \%$ Mujeres $38.6 \%$

\begin{tabular}{|c|c|c|c|}
\hline $\begin{array}{l}\text { Población } \\
\text { Económicamente } \\
\text { Activa (PEA) }\end{array}$ & Total & Hombres & Mujeres \\
\hline Ocupados & $54,152,266$ & $33,269,295$ & $20,883,061$ \\
\hline Desocupados & $1,886,205$ & $1,126,521$ & 759,684 \\
\hline
\end{tabular}

$3.3 \%$ Tasa de desocupación

$6.1 \%$ Tasa de desocupación de la población joven ( 18 a 29 años)

$46 \%$ de los ocupados tiene entre 30 y 49 años

Ocupados por posición en el trabajo

\begin{tabular}{|l|l|}
\hline Trabajadores asalariados & $68 \%$ \\
\hline Trabajadores por cuenta propia & $23 \%$ \\
\hline Empleadores & $5 \%$ \\
\hline Trabajadores sin pago & $4 \%$ \\
\hline
\end{tabular}
Críticas de Ocupación $19.3 \%$

* Nivel de escolaridad de la Población Desocupada $34 \%$ nivel primaria $30 \%$ nivel superior
La principales ramas en las que se concentran los ocupados:

$\checkmark$ Comercio

$\checkmark$ Industria manufacturera

$\checkmark$ Actividades agropecuarias

$\checkmark$ Otros servicios La Tasa de Informalidad Laboral es de $56.9 \%$

\section{OCUPADOS POR NIVEL DE EDUCACIÓN}

\begin{tabular}{|l|c|c|c|c|}
\hline & Hombres & Mujeres & $\begin{array}{c}\% \text { de } \\
\text { hombres }\end{array}$ & $\begin{array}{c}\% \text { de } \\
\text { mujeres }\end{array}$ \\
\hline $\begin{array}{l}\text { Sin } \\
\text { instrucción }\end{array}$ & $1,139,931$ & 696,590 & 62.1 & 37.9 \\
\hline Primaria & $8,425,928$ & $4,416,700$ & 65.6 & 34.4 \\
\hline $\begin{array}{l}\text { Secundaria o } \\
\text { media } \\
\text { superior }\end{array}$ & $17,206,397$ & $10,539,792$ & 62.0 & 38.0 \\
\hline Superior & $6,465,596$ & $5,209,868$ & 55.4 & 44.6 \\
\hline
\end{tabular}

$40 \%$ de la población desocupada tiene entre 20 y 29 años

$36 \%$ de la población desocupada tiene entre 50 y 59 años

"Incluye a las personas que se encuentran trabajando menos de 35 horas a la semana por razones ajenas a sus decisiones, más las que trabajan más de 35 horas semanales con ingresos mensuales inferiores al salario mínimo y las que laboran más de 48 horas semanales ganando hasta dos salarios minimos.

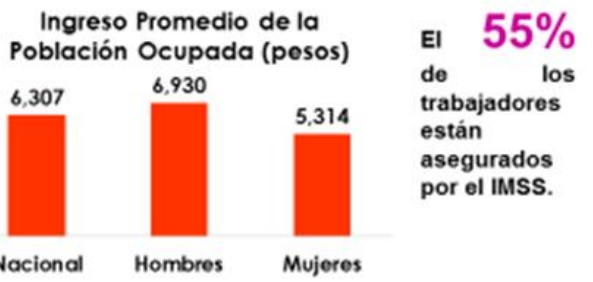

Figura 1. Condiciones laborales de la Población Económicamente Activa en México, segundo trimestre de 2019.

Fuente: Elaboración propia a partir de la Secretaría del Trabajo y Previsión Social, Información Laboral, segundo trimestre de 2019 
Población Económicamente Activa Femenina

$21,642,745$

Ocupadas

$20,883,061$

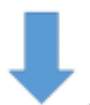

Tasa Neta de participación femenina: $43.7 \%$

Tasa Neta de participación masculina: $76.9 \%$

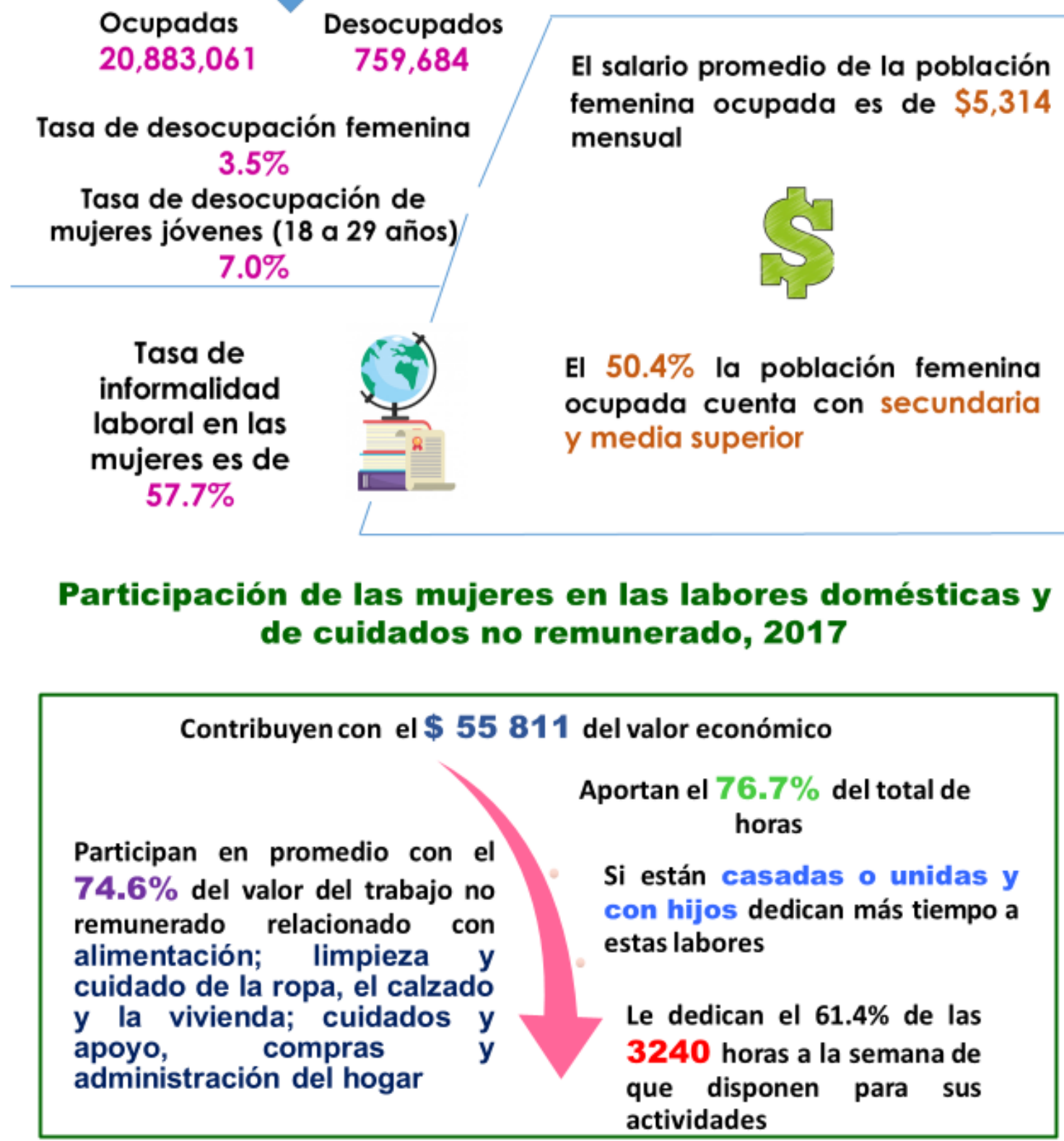

Figura 2. Condiciones laborales de las mujeres en México y su participación en las labores domésticas no remuneradas. 
Fuente: Elaboración propia a partir de la Secretaría del Trabajo y Previsión Social, Información Laboral, segundo trimestre de 2019; INEGI, 2017.

Social e históricamente a las mujeres se les ha impuesto la responsabilidad del trabajo doméstico, lo cual tiene dos efectos en su incorporación al mercado de trabajo, en primer lugar, disponen de menos tiempo para dedicarlo al trabajo remunerado y en segundo, las actividades de cuidado del hogar se trasladan cada vez más al ámbito de las actividades productivas. Las desigualdades de género que prevalecen en los mercados laborales de Latinoamérica provocan que las mujeres se ubiquen en trabajos relacionados con el comercio y los servicios relacionados con lo doméstico como son los cuidados, alojamiento y elaboración de comida, a medio tiempo y con salarios bajos. Estos roles tienen un efecto negativo en sus expectativas profesionales (OIT, 2016). Cabe mencionar que las mujeres de bajos recursos son las que tienen mayores problemas para insertarse al trabajo remunerado porque les es más complicado delegar a alguien más las responsabilidades que tienen en los quehaceres del hogar.

En América Latina las encuestas realizadas en las últimas décadas revelan que en promedio, las mujeres destinan dos tercios de su tiempo en el trabajo doméstico no remunerado y un tercio al trabajo pagado, mientras que los hombres ocupan su tiempo en la relación contraria. Además de la información que brindan las mediciones sobre el uso del tiempo, las encuestas de hogares de la región muestran que, en promedio, el 43,4\% de las mujeres de entre 20 y 59 años identifican razones familiares (embarazo, cuidado de niños y niñas o personas dependientes, trabajo doméstico o la prohibición por parte de miembros del hogar) como motivo principal para no buscar activamente o desempeñar un trabajo remunerado (CEPAL, 2017). La contribución de las mujeres en la reproducción de la fuerza de trabajo sigue siendo de suma importancia para el funcionamiento del sistema económico, sin embargo es una labor que no es reconocida, ni la que se realiza dentro del hogar y sin remuneración ni la que es remunerada.

\section{Emprendimiento femenino en México}

En relación a la actividad emprendedora de las mujeres, se observa que para 2019 de los 2.6 millones de personas que deciden iniciar un negocio más de 500 mil son mujeres, de las cuales el 75.2\% tiene entre 30 y 59 años de edad. Las principales características de las emprendedoras muestran que aquellas féminas que están casadas o en unión libre, son conyugues del jefe del hogar y tienen hijos, son más proclives a comenzar un negocio, el tamaño de los mismos es 
micro y se encuentran tanto en el sector formal como informal, además el $42.5 \%$ de las emprendedora tienen nivel medio superior y superior de estudios y es en las zonas urbanas donde existe mayor emprendimiento femenino.

La jornada de las emprendedoras es menor a la de los hombres con esta condición, y esto tiene su explicación en que ellas reparten su tiempo entre atender su empresa y su familia, las necesidades del hogar determinan la forma en que ellas se incorporan al mercado de trabajo. En el país el emprendimiento femenino es resultado de la búsqueda de opciones para hacer frente a las necesidades económicas que tienen en sus hogares, la mayoría de las mujeres buscan completar el ingreso que sus parejas obtienen, (figura 3), (INMUJERES, 2019).

Al iniciar un negocio ellas pueden hacer uso de los recursos materiales intelectuales que tengan y manejar su tiempo de manera más flexible permitiéndoles seguir atendiendo las labores domésticas, que como ya se ha mencionado estas actividades siguen siendo parte de las responsabilidades la población femenina.

Las mujeres empresarias siguen padeciendo la brecha de género, la apertura y la interdependencia de los mercados que ha generado la globalización no ha significado una mayor posibilidad de crecimiento internacional de las empresas creadas por mujeres, ellas tiene obstáculos para financiarse y fusionarse con otras (OCDE, 2018).

En cuanto a la compatibilidad de su rol como empresarias y responsables del hogar, por el número de horas que dedican a la actividad empresarial, el sacrificio es enorme para atender los oficios de la casa y el cuidado de sus hijos, sin que les quede mucho tiempo para otras actividades (ocio creativo, dedicación a la lectura, viajes, deporte, vida social y compartir con su cónyuge, entre otras), (Berdugo \& Gutiérrez, 2015:169).

\section{Elementos que impulsan el emprendimiento femenino}

La revisión bibliográfica y el análisis de los datos estadísticos permitió identificar algunos de los factores más relevantes que contribuyen a la creación y permanencia de nuevos negocios liderados por mujeres, es importante que los actividades y esfuerzos de las políticas públicas conozcan y reconozcan las condiciones, necesidades y motivaciones que la población femenina tiene y que la impulsan a crear sus propias empresas esperando que lo estos emprendimientos puedan sobrevivir y crecer a través del tiempo, para que tengan un verdadero impacto en el crecimiento y desarrollo del país. 


\section{6 millones de personas de 15 años y más en el mercado son empleadores/as.}

\section{6 mil son mujeres emprendedoras}

$7.8 \%$

De 15 a 29

años
$75.2 \%$

Entre 30 y 59

años
$16.9 \%$

Más de 60

años

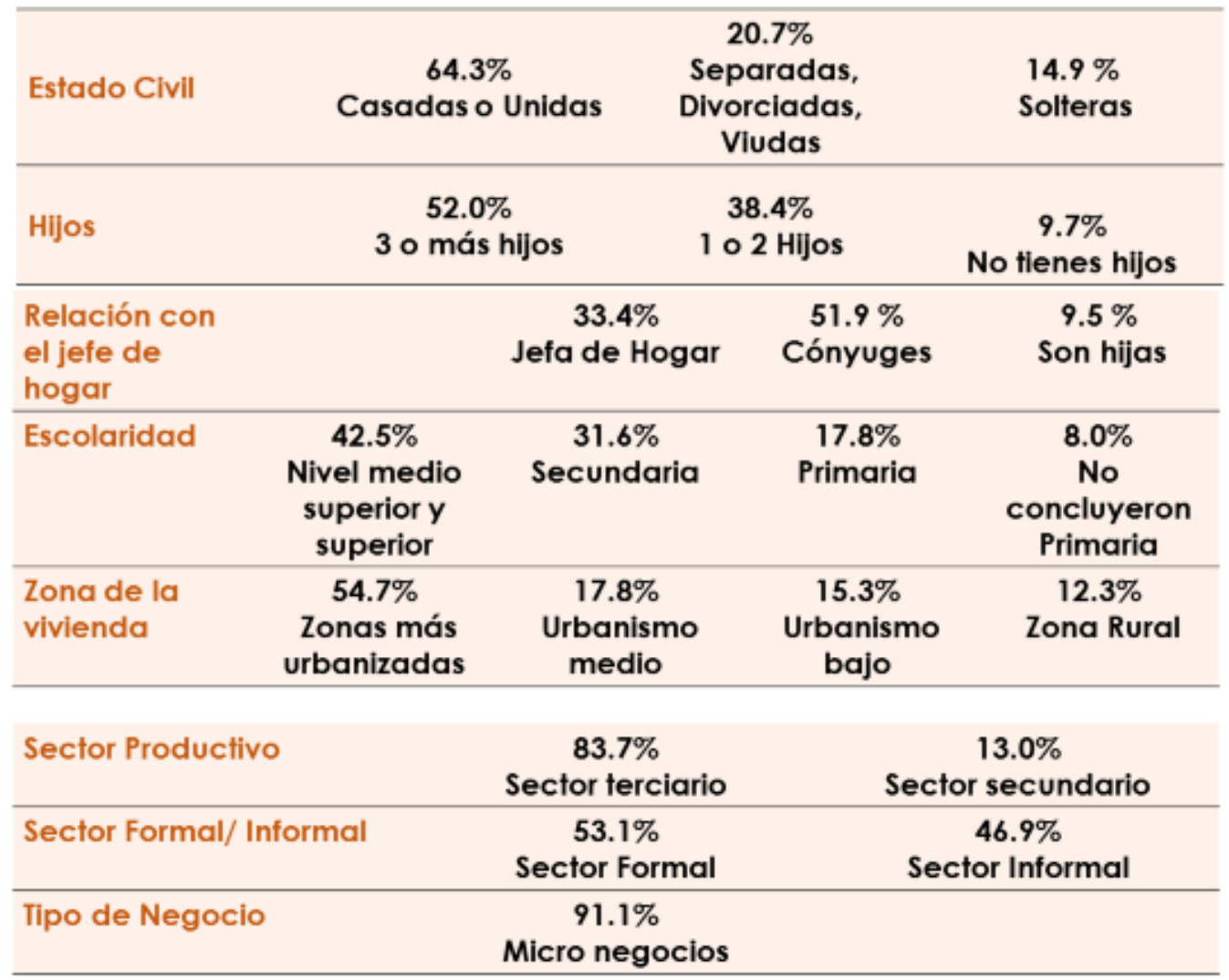

El Nivel de Ingresos es menor en las Mujeres Empleadoras que en los Hombres Empleadores

La Jornada Laboral de las Mujeres Empleadoras es menor al de los Hombres Empleadores

Figura 3. Características de las mujeres emprendedoras en México, 2019

Fuente: Elaboración propia a partir de INMUJERES, 2019 


\section{1) Acceso a financiamiento}

Dentro de las barreras para el emprendimiento, destacan las dificultades para conseguir financiamiento, "los intereses son muy altos, si de financiamiento se refiere, los impuestos y las obligaciones laborales constituyen una pesada labor para el emprendedor" (Amaru, 2008:7). El sistema financiero en el país tiene características que afectan particularmente a las mujeres, en primer lugar los bancos se han concentrado en otorgar créditos para el consumo, en segundo, excluyen a varios sectores de la población, a las pequeñas empresas y a personas con bajos ingresos, además los servicios financieros resultan costosos, debido a las altas tasas de interés y elevadas comisiones que cobran, esto agudiza las dificultades para obtener préstamos para la población en general y para las mujeres en particular, principalmente aquellas que requieren de recursos para iniciar o desarrollar sus empresas, (OCDE, 2018; CEPAL, 2016).

Resulta imperativa la regulación financiera por parte de la autoridades gubernamentales que permita el acceso al financiamiento a las mujeres emprendedoras, además conocer la realidad de dichas mujeres ayudará al diseño de políticas públicas encaminadas a este fin, es necesario que las emprendedoras puedan recibir capacitación en todo lo relacionado con los servicios financieros, el ahorro, las inversiones, el riesgo, las tecnologías digitales, entre otros temas vinculados con las administración y finanzas de los negocios. Esto contribuirá para que la esperanza de vida de los emprendimientos se amplié, comúnmente los recursos que utilizan para crear los negocios son propios o buscan préstamos o socios con gente cercana como amigos o familiares, lo cual reduce el margen en la toma de decisiones, ya que estos recursos regularmente son pequeños. En pocas palabras se debe impulsar la profesionalización de estas empresas.

\section{2) Habilidades y conocimientos}

Las propuestas o programas de emprendimiento para las mujeres deben estar relacionadas con su cultura, su cotidianidad y con los recursos materiales y naturales con lo que cuenten (Maldonado, Lara \& Maya, 2016; Alvares, 2013).

Mora, Meli \& Astete, (2018:50) realizaron una investigación sobre las experiencias de participar en emprendimientos de mujeres mapuche en Chile y de mujeres mayas en México, las primeras indicaron que los programas gubernamentales de emprendimiento en muchas ocasiones no se vinculan con su vida cotidiana ni con sus conocimientos, ellas por ejemplo se denominan gente de mar, por lo que sus interés se encuentran en la explotación de productos marinos, tejidos con telar o productos afines con sus conocimientos medicinales, no obstante les ofrecieron 
emprendimientos relacionados con la crianza de ovinos, actividad que tuvieron que aceptar. En el saco de las segundas, las mujeres mayas, también reconocen las habilidades que tienen para coser, bordar y trabajar la tierra como parte de sus conocimientos ancestrales, los que pueden ser utilizados en los emprendimientos.

Se debe hacer el esfuerzo, principalmente en el caso de mujeres de comunidades rurales, de que los programas de impulso a emprendimientos consideren los intereses, inquietudes $\mathrm{y}$ conocimientos que las mujeres tengan y que las motive a participar en la creación de empresas, es cierto que esto es un tema complejo porque no se trata solo de producir, la parte de comercialización es muy importante, lo que se produzca debe tener demandan, hacer compatibles estos puntos, es tarea de la política pública.

\section{3) Acompañamiento}

El impulso de emprendimientos productivos de mujeres, principalmente de aquellas que se encuentran en una situación socioeconómica precaria debe provenir de aliados estratégicos como los llama Alvares (2013), que es una persona que tenga como característica principal el liderazgo, elemento fundamental para hacer posible la creación de un negocio. Para muchas mujeres es difícil dar el primer paso para la creación de una empresa, la falta de confianza, la educación insuficiente, el exceso de trámites y gestiones a realizar, la escasez de dinero y la demanda de tiempo que se invierte en las labores de casa, son una serie de obstáculos que enfrentan, por ello es necesaria la capacitación continua y el acompañamiento durante el tiempo que sea necesario para el nacimiento y la madurez del emprendimiento, para que después puedan continuar ellas solas, (Rodríguez, 2013; Cisneros, Meza \& Meza, 2016; Maldonado, Lara \& Maya, 2016).

Robinson, Díaz-Carrión \& Cruz, (2019:103), indican que "el acompañamiento requiere centrarse en la empatía, la capacitación constante y el logro equilibrado de avances en las dimensiones consideradas en el empoderamiento: económica, personal, familiar y sociocultural, en la esfera pública y privada".

Por lo tanto, la gestación y desarrollo de emprendimientos productivos deben estar encabezados por aliados estratégicos que podría ser algún representante de las autoridades locales, alguna persona física o moral, organizaciones civiles o representantes de la iglesia, (Alvares, 2013), que puedan guiar o encaminar a las mujeres para que tomen la decisión de emprender. 


\section{4) Entorno económico y programas públicos}

El apoyo de programas públicos y de instituciones gubernamentales es un elemento importante para emprender y desarrollar un nuevo negocio, uno de los retos más grandes para los micro negocios liderados por mujeres es que en el largo plazo puedan sobrevivir y fortalecerse, en esto los programas públicos juegan un papel importante al otorgarles no solo financiamiento, sino apoyo en diversos ámbitos y áreas relacionadas con el negocio y con el empoderamiento de las empresarias, aspectos que van desde cuestiones administrativas y toma de decisiones hasta aquellos relacionados con la autoestimada de la empresarias, entre muchas otras cosas.

Una situación que se olvida en el diseño y aplicación de los programas públicos es que los procedimientos, trámites y obligaciones que deben cumplirse para poder participar en las convocatorias sean perfectamente comprensibles para la población objetivo. En algunas ocasiones se pide que se elabore un plan de negocio y ya se ha dicho que los emprendimientos son micro negocios que en su mayoría están en la informalidad y sus dueñas tienen bajo nivel educativo, entonces es innegable que será poco fácil que puedan cumplir con todos los requisitos que se piden para participar en algún programa gubernamental y no necesariamente porque no los tengas, simplemente porque no saben cómo hacerlo.

Rodríguez, Baños, Saiz, Valencia, Muñiz, \& Flores, (2017:52) realizaron un estudio sobre el ecosistema de emprendimiento en Jalisco, México, aplicando la metodología del Monitor Global de Emprendimiento (GEM), encontraron, entre otras cosas que los apoyos que otorga el gobierno a las empresas van destinados a negocios que tengan un mínimo de años en operación, excluyendo a los emprendimiento que apenas comienzan y a esto se le sema que los trámites administrativos para participar en las convocatorias para obtener recursos públicos son demasiado burocráticos.

Las políticas públicas que estén destinadas a apoyar e impulsar el emprendimiento femenino deben buscar subsanar desventajas, apoyar su crecimiento, aminorar los efectos negativos de los cambios en las variables que escapan al control de las empresarias, para que esto sea posible es necesario conocer los problemas reales que enfrentan las mujeres en el mercado de trabajo y en su cotidianidad social, cultural, económica y política.

Una vez que se conocen las problemáticas que enfrenta el emprendimiento femenino es posible diseñar soluciones e identificar áreas de oportunidad, donde la política pública puede actuar 
siendo reactiva, proponiendo soluciones a las necesidades, y proactiva, al definir objetivos claros de conducción de la evolución en la creación de empresas lideradas por mujeres.

Además, el impulso al emprendimiento no solo femenino, en general de la población, requiere que el diseño de las políticas públicas este enmarcado dentro del entorno económico nacional e internacional, que es cada vez más complejo e incierto, y que los países en desarrollo son cada vez más vulnerables ante los cambios de este entorno, por tal motivo es imperativo que se haga un esfuerzo por establecer un vínculo entre las necesidades de estabilidad macroeconómica y los requerimientos de las emprendedores. El entorno económico se define como el conjunto de variables macroeconómicas, inflación, tasas de interés, tipo de cambio, precios internacionales, cuyo comportamiento crea escenarios concretos de corto plazo que afectan el ahorro, la inversión, la balanza de pagos, las reservas internacionales, el empleo, el PIB, la demanda agregada, y todo ello impacta de manera importante en el desarrollo y crecimiento de un país y por lo tanto en la creación, permanencia y crecimiento de nuevas empresas.

A los diferentes agentes económicos, las autoridades (en sus diferentes niveles) deben proveerle escenarios con los mayores niveles de certidumbre, para que ellos puedan enfrentar no solo el presente inmediato, también para que sean capaces de adelantarse al futuro próximo, de tal manera, que los efectos adversos de desequilibrios nacionales e internacionales no causen en ellos estragos mayores.

\section{REFLEXIONES FINALES}

Este trabajo muestra que es imperativo fortalecer la capacidad emprendedora de las mujeres, facilitándoles información, intercambio de experiencias, capacitándolas para la creación de alianzas comerciales, dándoles asesoría técnica y administrativa para la gestación y desarrollo de negocios.

La información, seguimiento y análisis continuo de los resultados del emprendimiento femenino deben ser también elementos que permitan la continua retroalimentación y evaluación de las políticas públicas orientadas a tal fin y de su valor añadido.

Las mujeres cuentan con habilidades y conocimientos que han sido adquiridas a lo largo de su vida de manera formal e informal, las cuales pueden convertirse en capacidades productivas aunadas a los recursos monetarios y materiales que pueden tener, lo que representa una combinación que puede dar pie al nacimiento de un emprendimiento que permita generar 
autoempleo y empleo, lo cual puede tener un efecto positivo en el desarrollo de una región o comunidad, para ello es necesario que se apoye a las emprendedoras pero mirando el contexto real en el que viven.

Los emprendimientos femeninos debe surgir en un entorno que los encamine a la profesionalización de los mismos, considerando que muchas de las empresarias necesitan ser acompañadas en diversas áreas de su quehacer empresarial y personal, este último elemento es relevante porque implica encaminar a las mujeres hacia el empoderamiento, lo que les permitirá ser más autónomas y seguras en la toma de decisiones.

En el diseño y aplicación de las políticas públicas encaminadas a apoyar el emprendimiento de las mujeres, se deben considerar las condiciones en las que se encuentra la población femenina a la que se quiera motivar para emprender. Además, con respecto a las mujeres que ya son empresarias, se olvida analizar las motivaciones que hayan tenido para iniciar su propio negocio, esto permitirá que los programas destinados a ellas tengan mayores efectos positivos en el desarrollo y esperanza de vida de sus negocios.

Pero tal parece que para los gobiernos en turno es más fácil otorgar pequeños apoyos para crear micro negocios, muchos de ellos en la informalidad y con escasas probabilidades de sobrevivencia, sin embargo esto solo tiene mínimos efectos en el largo plazo.

Por último, cabe mencionar que los estudios sobre emprendimiento femenino deben profundizarse y abordarse de manera multidimensional incorporando aspectos como género, generación, actividad económica, clase social, entre otros, que permitan tener una visión más clara y completa sobre su compleja realidad. No se puede seguir hablando de emprendimiento, diciendo que a través de él se pueden generar empleos y crecimiento, si no hay un verdadero compromiso por parte de las instituciones gubernamentales encargadas de impulsarlo. Hoy más que nunca la generación de empleos formales y bien remunerados sigue estando en la agenda pública, el emprendimiento es una opción pero debe asumirse dentro de las condiciones reales que vive la población trabajadora en el país, especialmente las mujeres.

Se trata de que se combine el mercado con un Estado que sea capaz de promover una economía solidaría, para ello es necesario que se establezcan, por un lado, los límites de la intervención del Estado en la actividad económica y, por otro lado, se regule el mercado. 


\section{REFERENCIAS}

(1) Alvares, C. (2013). Emprendimientos productivos para la generación de empleo, mujeres del municipio de El Corpus, Departamento de Choluteca, Honduras. Tesis de maestría. Universidad Nacional Autónoma de Honduras, Tegucigalpa, Honduras. [En línea] Disponible en: https://tzibalnaah.unah.edu.hn/bitstream/handle/123456789/3380/T-MSc00123.pdf?sequence=2. recuperado: 5 de agosto de 2019.

(2) Amaru, A. (2008). Administración para emprendedores, Fundamentos para la creación y gestión de nuevos negocios. México: Pearson. 241 p.

(3) Berdugo, E; \& Gutiérrez, J. (2015). "Mujeres empresarias de Bogotá". En: Revista CIFE: Lecturas de Economía Social, Vol. 17, no, 26. pp. 149-174.

(4) Canales, R; Román, Y; \& Ovando, W. (2017). "Emprendimiento de la población joven en México. Una perspectiva crítica". En: Entreciencias: Diálogos en la sociedad del conocimiento, vol. 5, no 12. México: UNAM. Pp. 1-17. [En línea] Disponible en: http://www.redalyc.org/articulo.oa?id=457650040001 recuperado: 6 de julio de 2019.

(5) Cisneros, L; Meza, T; \& Meza, J. (2016). "Análisis de los microemprendimientos dirigidos por mujeres en un sector popular de la ciudad de Guayaquil- Ecuador". En Revista Observatorio de la Economía Latinoamericana, no. 224. Ecuador, [En línea] Disponible en: http://www.eumed.net/cursecon/ecolat/ec/2016/mujeres.html recuperado: 3 de septiembre de 2019.

(6) Comisión Económica para América Latina y el Caribe. CEPAL. (2018). La ineficiencia de la desigualdad (LC/SES.37/3-P), Santiago, Chile.

(7) Comisión Económica para América Latina y el Caribe. CEPAL. (2018). Agenda 2030 y los Objetivos de Desarrollo Sostenible: una oportunidad para América Latina y el Caribe (LC/G.2681/Rev.2), Santiago, Chile.

(8) Comisión Económica para América Latina y el Caribe. CEPAL. (2016). Autonomía de las mujeres e igualdad en la agenda de desarrollo sostenible (LC/G.2686/ Rev.1 - LC/G.2686(CRM.13/3)), Santiago, Chile.

(9) Comisión Económica para América Latina y el Caribe. CEPAL. (2017). Panorama Social de América Latina. (LC/PUB.2017/12-P), Santiago, Chile. 
(10) Hernández, R; Fernández, C; \& Baptista, P. (2006). Metodología de la investigación. Editorial McGraw-Hill Interamericana, México DF. México.

(11) Instituto Nacional de Geografía Estadística e Informática. INEGI. (2017). Cuenta satélite del trabajo no remunerado de los hogares de México. INEGI, México. [En línea] Disponible en: https://www.inegi.org.mx/contenidos/saladeprensa/boletines/2018/StmaCntaNal/CSTNRH2017.pdf recuperado: 23 de octubre de 2019.

(12) Instituto Nacional de Geografía Estadística e Informática. INEGI. (2019). Resultado de la encuesta nacional de ocupación y empleo, cifras durante el segundo trimestre de 2019. Comunicado de prensa Núm. 399/19. México https://www.inegi.org.mx/contenidos/saladeprensa/boletines/2019/enoe_ie/enoe_ie2019_08.pdf

(13) Instituto Nacional de las Mujeres. INMUJERES. (2019). Empleadoras. [En línea] Disponible en: http://estadistica.inmujeres.gob.mx/formas/tarjetas/Empleadoras.pdf recuperado: 1 de mayo de 2019.

(14) Maldonado, B; Lara, G; \& Maya, A. (2016). "La mujer como motor del desarrollo local: una experiencia puntual”. En: Revista San Gregorio, Número especial sobre el desarrollo local, ISSN 1390-7247, pp. 91-107.

(15) Mora, G; Meli, D; \& Astete, P. (2018). "Empoderamiento y demanda de autogestión. Estudio comparativo de emprendimientos de mujeres indígenas". En: Sophia Austral, no 21, Pp. 43-59.

(16) MUJERES, ONU. (2016). Empresas y empoderamiento de las mujeres. Principios para el empoderamiento de las mujeres en las empresas. Ciudad de México, México. [En línea] Disponible en: http://www.onu.org.mx/wp-content/uploads/2016/12/Empoderamiento-mujeres-en-empresas.pdf recuperado: 10 de octubre de 2019.

(17) Organización de Cooperación y Desarrollo Económico. OCDE. (2018). "Empowering Women in the Digital Age. Where we stand?” (High-Level Event), OECD, marzo. Estados Unidos.

(18) Organización Internacional del Trabajo. OIT. (2016). Las mujeres en el trabajo, tendencias Enero, Ginebra, Suiza.

(19) Pierre, A; \& Molina, R. (2012). Una teoría sobre el emprendimiento regional en la economía del conocimiento, México: Pearson. 
(20) Robinson, D; Díaz-Carrión, I; \& Cruz, S. (2019). "Empoderamiento de la mujer rural e indígena en México a través de grupos productivos y microempresas sociales". En: RETOS. Revista de Ciencias de la Administración y Economía, vol. 9, no 17, Pp.91-108 https://10.17163/ret.n17.2019.06

(21) Rodríguez, B. (2013). Propuesta de merchandising para tiendas de autoservicios locales de la Ciudad de Huajupan de León, Oaxaca. (Tesis inédita de licenciatura) Universidad Tecnológica de la Mixteca, Huajuapan de León, Oaxaca.

(22) Rodríguez, L; Baños, V; Saiz, J; Valencia, R; Muñiz, E; \& Flores, M. (2017). Monitor Global de Emprendimiento (GEM) Jalisco 2016/2017: Ecosistema de emprendimiento en la Zona Metropolitana de Guadalajara. Zapopan, Jal. (México): Tecnológico de Monterrey, Campus Guadalajara. http://gda.itesm.mx/gemjalisco/GEM_Jalisco_2016-2017.pdf

(23) Ruiz, N; Camelo O; \& Franco, L. (2015). Emprender con talento: Mujer y emprendimiento estratégico en Emprendimiento femenino en Iberoamérica, coordinador Senén Barro Ameneiro, Redemprendia, España.

(24) Secretaria del Trabajo y Previsión Social. STPS. (2019). Información laboral, junio 2019 México

(25) Vallmitjana, P. (2014). La actividad emprendedora de los graduados IQS. 2014. Tesis Doctoral. Universitat Ramon Llull. $\quad$ [En línea $]$ Disponible https://www.tdx.cat/bitstream/handle/10803/145034/Tesis\%20Nuria\%20Vallmitjana\%20Palau.pdf?sequence $=1$ recuperado: 30 de agosto de 2019 .

(26) Valls, J; Cruz, C; Torruella, A; Juanes, E; Canessa, M; \& Hormiga, E. (2012). Causa del fracaso de los

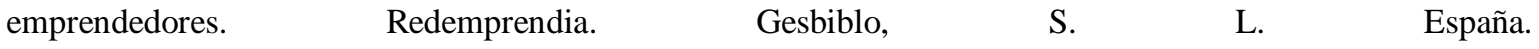
https://www.redemprendia.org/sites/default/files/descargas/causas_de_fracaso_de_los_emprendedores.pdf

(27) Viadana, C; Zubeldía, M; Tomarelli, J; \& Morales, O. (2016). Mujeres emprendedoras: una aproximación empírica. Vigesimoprimeras Jornadas: Investigaciones en la Facultad de Ciencias Económicas y Estadística. Noviembre. Universidad del rosario. [En línea] Disponible en: https://www.fcecon.unr.edu.ar/webnueva/sites/default/files/u16/Decimocuartas/viadana_zubeldia_y_otros_mujeres_emprendedoras.pdf recuperado en: 5 de junio de 2019. 